\title{
Nonmagnetic metamaterial landscapes for guided electromagnetic waves
}

\author{
S. Viaene*a,b, V. Ginis ${ }^{\mathrm{a}}$, J. Danckaert ${ }^{\mathrm{a}}$, P. Tassin ${ }^{\mathrm{b}, \mathrm{a}}$ \\ ${ }^{a}$ Applied Physics Research Group, Vrije Universiteit Brussel, Pleinlaan 2, B-1050, Brussel, \\ Belgium; \\ ${ }^{b}$ Department of Physics, Chalmers University of Technology, SE-412 96, Göteborg, Sweden.
}

\begin{abstract}
Transformation optics provides a geometry-based tool to create new components taking advantage of artificial metamaterials with optical properties that are not available in nature. Unfortunately, although guided electromagnetic waves are crucial for optical circuitry, transformation optics is not yet compatible with two-dimensional slab waveguides. Indeed, after determining the propagation of confined waves along the waveguide with a two-dimensional coordinate transformation, the conventional application of transformation optics results in metamaterials whose properties are insensitive to the coordinate perpendicular to the waveguide, leading to bulky, and therefore impractical, designs. In this contribution, we formulate an alternative framework that leads to feasible coordinate-based designs of two-dimensional waveguides. To this end, we characterize a guided transverse-magnetic light mode by relevant electromagnetic equations: a Helmholtz equation to account for wave propagation and a dispersion relation to impose a continuous light profile at the interface. By considering how two-dimensional conformal transformations transform these equations, we are able to materialize the coordinate-designed flows with a nonmagnetic metamaterial core of varying thickness, obtaining a twodimensional device. We numerically demonstrate the effectiveness and versatility of our equivalence relations with three crucial functionalities, a beam bender, a beam splitter and a conformal lens, on a qualitative and quantitative level, by respectively comparing the electromagnetic fields inside and the transmission of our two-dimensional metamaterial devices to that of their three-dimensional counterparts at telecom wavelengths. As a result, we envision that one coordinate-based multifunctional waveguide component may seamlessly split and bend light beams on the landscape of an optical chip.
\end{abstract}

Keywords: Metamaterial waveguides, Transformation optics, Guided electromagnetic waves, Dispersion engineering

\section{INTRODUCTION}

The development of structured artificial materials, e.g., metamaterials with subwavelength structures [1-3], has opened up a wealth of optical properties that may now be used to overcome fundamental challenges in photonics. One of these challenges consists of the long-range manipulation of confined light flows along two-dimensional material systems. Such light flows are indispensable to applications in integrated optics, e.g., (bio-)sensing [4-5], optical circuitry [6-7], and alloptical device actuation [8-9]. In particular, these applications benefit from the strong confinement of light, which is currently achieved either by making use of bandgap materials [10-12] or by coupling to plasmon polaritons on metaldielectric and graphene-dielectric interfaces [13-14]. The latter metal- and graphene-dielectric interfaces long seemed to be preferred material platforms, especially due to their similarity to electronic circuits which allows them to be fabricated with existing lithography techniques.

Unfortunately, any metal-dielectric waveguide suffers from a trade-off between the confinement of light and its propagation range due to dissipation loss, especially at infrared and optical frequencies [15]. To remedy the metallic losses that are inherent to metal-dielectric interfaces, researchers have turned to dielectric-dielectric (metamaterial) interfaces, making use of phonon polaritons sustained by polar dielectrics [16], Dyakonov surface waves along anisotropic dielectric interfaces [17] and mie-resonance based metamaterials or metasurfaces [18-20]. In recent years, the scope of all-dielectric metamaterials has systematically increased, e.g., dielectric building blocks may now sustain both positive and negative permittivity and permeability distributions, and fabrication tools such as direct laser writing with femtosecond pulses [2122] and self-assembly [23] allow for large-area and highly anisotropic metamaterial designs.

*sophie.viaene@vub.ac.be

Metamaterials, Metadevices, and Metasystems 2016, edited by Nader Engheta,

Mikhail A. Noginov, Nikolay I. Zheludev, Proc. of SPIE Vol. 9918, 99180X

(C) 2016 SPIE - CCC code: 0277-786X/16/\$18 - doi: 10.1117/12.2238502 


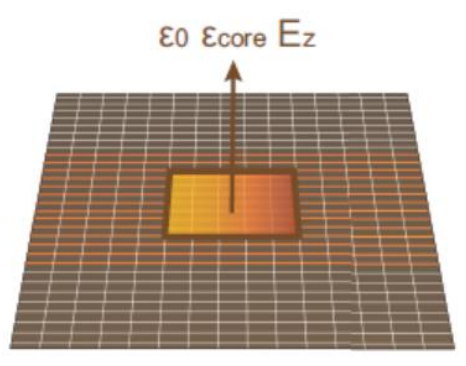

$(u, v)$
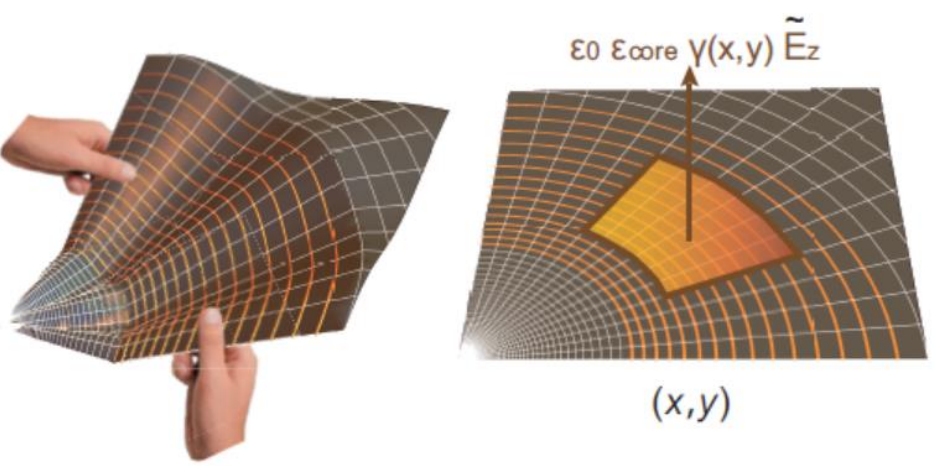

Figure 1. To manipulate confined light flows along the symmetry plane of a slab waveguide (a), we locally stretch the symmetry plane with a two-dimensional conformal coordinate transformation (b) so that light bends over 90 degrees as if it experiences a geometry g\| $=$ $\gamma(x, y)$ (c). The metamaterial implementation of the transformation makes sure that the dielectric flux through the symmetry plane is identical to that in the transformed waveguide with nontrivial geometry.

Given the diversity of all-dielectric metamaterials and their associated optical properties, we are in need for an effective design tool that relates the properties of artificial dielectric waveguides to a desired functionality. In this contribution, we discuss the design of metamaterial waveguides with an anisotropic metamaterial core of varying thickness based on twodimensional coordinate transformations of confined light flows [24-25]. In particular, we provide two relations-a relation defining the dielectric properties of the core and a relation defining the thickness variation-that allow imposing particular light trajectories along the optical landscape of a dielectric waveguide with moderate confinement [24].

The outline of this paper is as follows: In Sec. 2, we introduce concept of coordinate-based metamaterials according to the existing framework of transformation optics. In Sec. 3, we focus on the manipulation of guided modes propagating along the symmetry plane of a dielectric slab waveguide, consisting of a high-index core layer $\epsilon_{\text {core }}$ and low-index outer layers $\epsilon_{\text {out }}$ such as vacuum. We notice that the straightforward application of transformation optics with two-dimensional coordinate transformations on the waveguide symmetry plane leads to bulky and impractical designs, with metamaterial implementations both inside and outside of the core region. To avoid bulky designs, we derive specific equivalence relations for guided electromagnetic modes, based on the effect of a two-dimensional coordinate transformation on their characteristic equations. Our equivalence relations lead to feasible and low-loss metamaterial waveguide cores of varying thickness without need of metamaterial implementations in the outer layers. In Sec. 4, we demonstrate the flexibility and effectiveness of our equivalence relations with numerical simulations of three proof-of-principle devices: a beam bender, a beam splitter and a conformal lens.

\section{COORDINATE-BASED MANIPULATION OF LIGHT}

To impose desired confined light flows in free space, researchers often rely on the framework of transformation optics [2630]. Transformation optics naturally extends the principle of Fermat, which relates the trajectories of light rays to variations in the dielectric properties of an optical system [31]. Essentially, transformation optics establishes an equivalence between the behavior of light rays in inhomogeneous and anisotropic electromagnetic media and the propagation of light through nontrivial space-times [32-34]. In general relativity light also propagates along trajectories corresponding to an extreme "optical path length", known as geodesics of a space-time geometry. Mathematically, the Maxwell equations on such a nontrivial space with geometry $g_{i j}$ are exactly imposed by a specific electromagnetic metamaterial if the material's electric (permittivity $\epsilon^{i j}$ ) and magnetic (permeability $\mu^{i j}$ ) material distributions satisfy analytical equivalence relations [26]

$$
\varepsilon^{i j}=\sqrt{g} g^{i j}=\mu^{i j} .
$$

These relations [Eq. (1)] ensure that light propagates inside a transformation-optical metamaterial as if it were moving through the (nontrivial) space.

Because the geometrical tools of transformation optics naturally extend Fermat's principle beyond the ray approximation [35], they allow exploring unexpected and exciting phenomena in anisotropic and inhomogeneous metamaterials. After impressive designs of invisibility cloaks [26-30], the geometrical understanding of the interaction of light with 
metamaterials has not only improved our control on the flow of light [36-37], the emission of electromagnetic radiation [38-39], and our understanding of effective gauges [40-41], but also allows to study the behavior of light in space-times that are actual solutions of the Einstein equations, such as artificial black holes [42-43] and the cosmological redshift [44].

The geometry of the nontrivial space $g_{i j}$ crucially determines the light flows and the associated functionality of the device. As shown in Fig. 1(a)-(c), one may apply a coordinate transformation to deform initially straight light flows until they conform to a desired set of light trajectories. Technically, the coordinate transformation transforms the trivial Euclidean geometry $\delta_{i j}$ in a covariant way until it obtains a coordinate-induced geometry $g_{i j}$, which may be expressed in terms of the Jacobian $\Lambda_{i}^{i^{\prime}}=\partial \mathrm{x}^{i^{\prime}} / \partial \mathrm{x}^{i}$ associated to the coordinate transformation from old (Euclidean) coordinates $x^{i^{\prime \prime}}$ to new coordinates $x^{i}[33]$

$$
g_{i j}=\Lambda_{\prime}^{i^{\prime}} \delta_{i^{\prime} j^{\prime}} \Lambda_{j}^{j^{\prime}},
$$

making use of the Einstein summation convention. The corresponding geodesics of the nontrivial geometry $g_{i j}$ lead to desired light flows which materialize inside an optical device with impedance-matched metamaterials based on the equivalence relations [Eq. (1)].

\section{TRANSFORMING GUIDED MODES}

In principle, the framework of transformation optics should also apply to light along lower-dimensional systems. Indeed, the existing framework of transformation optics [Eq. (1)] has also been successfully applied to single metal-dielectric interfaces [45-48] and graphene-dielectric interfaces [49-50]. In case of metal-based surface plasmons, transformation optics has no need for adaptations because the energy of surface waves, which are confined to a metal-dielectric interface, mostly reside inside the dielectric at frequencies far from the surface plasmon resonance of the metal. As a result, one may apply the conventional framework of transformation optics only to the dielectric layer to impose coordinate-based surface waves along the interface.

The application of transformation optics to guided modes along a slab dielectric waveguide, however, leads to bulky and impractical designs. If we deform confined light flows along the symmetry plane of the waveguide with a two-dimensional coordinate transformation, e.g., a logarithmic map implementing a beam bend [37] as shown in Fig. 1(a)-(c), the conventional equivalence relations of transformation optics [Eq. (1)] generate material distributions that are independent of the transverse coordinate. The resulting impedance-matched metamaterial implementations should, in principle, extend to infinity outside of the core layer, resulting in bulky and impractical designs. In this section, we analyze the effect of a two-dimensional coordinate transformation on the characteristic equations of a transverse-magnetic (TM) guided mode to come up with alternative and efficient equivalence relations, based upon the description of the guided mode in the reciprocal space [Fig 2(a)-(c)].

As a start, we have a look at the characteristic equations of a guided mode along an ordinary slab waveguide. An incident transverse-magnetic guided mode of angular frequency [Fig 2(a)] consists of a confined transverse profile that propagates along the symmetry plane with propagation constant $\beta$. The transverse profile is confined to the core layer and is both characterized by exponentially decaying tails outside of the core with extinction coefficient $k_{l}$ and by sinusoidal variations inside the core with standing wave number $k_{2}$ (Fig. $1 \mathrm{~g}$ ).

The reciprocal space of the guided mode $\left(\beta, k_{1}, k_{2}\right)$ will help us to construct our equivalence relations (Fig. 2). The characteristic equations of a guided mode, i.e., a Helmholtz wave equation and a dispersion relation, relate these components at a fixed frequency $\omega$. On the one hand, the Helmholtz equation of a guided TM-mode

$$
\left[\Delta_{x y} \pm k_{1,2}^{2}+\epsilon_{\text {core, out }} \frac{\omega^{2}}{c^{2}}\right] H_{||}=0 .
$$

imposes circular isofrequency contours inside the core and hyperbolic isofrequency contours outside of the core [Fig. 2(a)]. These contours are compatible for a range of propagation constants, within the green band. On the other hand, only one particular propagation constant $\beta(\omega)$, indicated by the green line in Fig. 2(a), will sustain a continuous transverse 
(a)

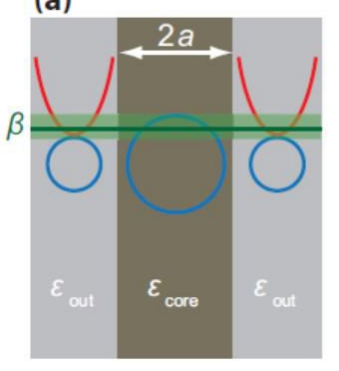

(b)

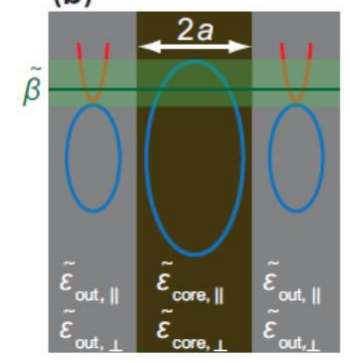

(c)

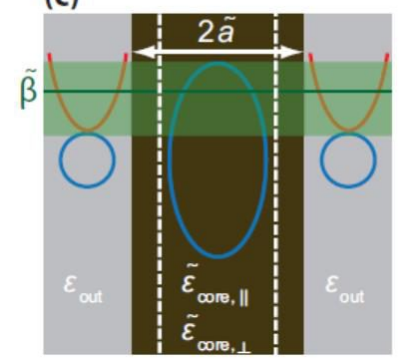

Figure 2. Our design uses a nonmagnetic uniaxial metamaterial waveguide of varying thickness to impose two-dimensional flows of light. We rely on the wave vector space of a guided mode to establish our equivalence relations., shown in (a) for a simple dielectric waveguide. (b) As a consequence of the in-plane transformation, the vector space of the incident guided mode with propagation constant $\beta$ (green) is stretched in the symmetry plane. The wave vectors lie respectively on elliptical (hyperbolic) isofrequency contours of the wave equation in blue (red) inside the core (outer) region. According to the traditional recipe of transformation optics, the exponential tails $\left(k_{l}\right)$ and thickness $a$ are preserved because metamaterials are implemented in the core and outer layers. (c) To preserve confinement and to impose a globally stretched propagation vector without metamaterials in the outer layers, a thickness variation $\tilde{a}$ ensures the continuity conditions at the interfaces at the expense of changes in the exponential tails $\left(k_{l}\right)$.

profile at the interfaces. The continuous profile on a waveguide of thickness $2 a$ is imposed by the dispersion relation of the TM-mode :

$$
\tan \left(k_{2}(\omega, \beta) a\right)=\frac{\epsilon_{\text {core }} k_{1}(\omega, \beta)}{\epsilon_{\text {out }} k_{2}(\omega, \beta)} .
$$

Now, we consider how the Helmholtz equation and the dispersion equation change due to a two-dimensional conformal transformation on the symmetry plane of the slab waveguide. A conformal transformation locally rescales all in-plane vectors with a stretching $X$ and rotates them without changing their relative angles [15, 51]. Equation (2) readily extracts the induced geometry, which is isotropic in the waveguide plane $\mathrm{g}_{\|}=\gamma(\mathrm{x}, \mathrm{y}) \delta_{\mathrm{ij}}$ and trivial perpendicular to it $g_{\perp}=1$. In Ref. [49], we impose the in-plane propagation with a metamaterial inside the core layer that reproduces the Helmholtz equation of the induced geometry, i.e., a unidirectional nonmagnetic core whose anisotropy is proportional to the induced geometry

$$
\epsilon_{\perp \text { core }, \text { out }}=\gamma(x, y) \epsilon_{\text {core }, \text { out }}, \epsilon_{\| \text {core }, \text { out }}=\epsilon_{\text {core }, \text { out }}, \mu=1 .
$$

As a consequence, the sinusoidal variations inside the core are preserved.

We have obtained the metamaterial implementation [Eq. (5)] in a mathematically rigorous way through a mapping of the covariantly transformed wave equation with $\gamma(x, y)$ to the wave-equation with arbitrary anisotropic dielectrics. In this contribution, however, we motivate the required material implementation for a guided TM-mode in an intuitive way. Since the metamaterial reproduces the behavior of the guided mode in the transformed space, it should in particular impose the same electromagnetic fields and fluxes. For example, the dielectric flux perpendicular to the symmetry plane contains the transformed electric field $\tilde{E}_{z}$ and a deformed surface element $d x d y$,

$$
\widetilde{D}_{z}=\int(\gamma(x, y) d x d y) \epsilon_{0} \epsilon_{\text {core }} \widetilde{E}_{z}(x, y) .
$$

To impose the same flux with a material instead of a geometry, one should account for the change in volume element. This is easily done by making the material anisotropic as in Eq. (5) [Figs. 1(c) and 2(c)]. Notice that, because we consider a transverse-magnetic mode, the magnetic flux is automatically preserved and requires no material implementation. Similarly, the in-plane dielectric components do not require any changes. 
(a)

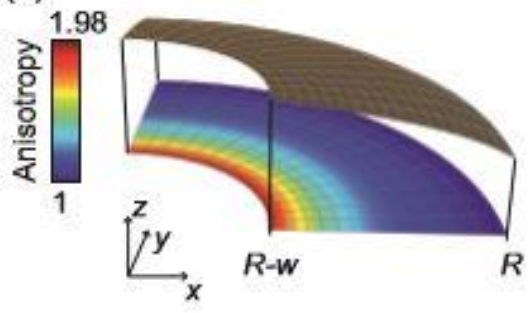

(b)

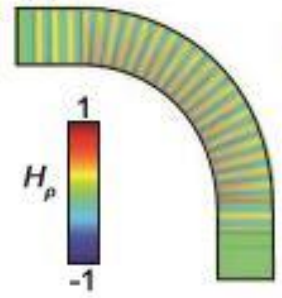

(c) (d)

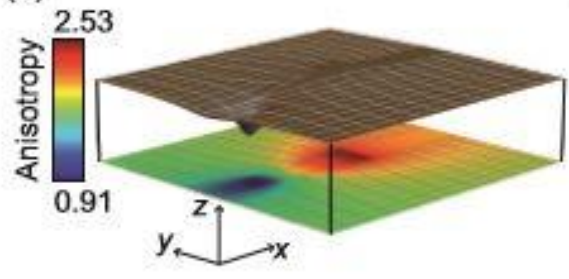

(e)

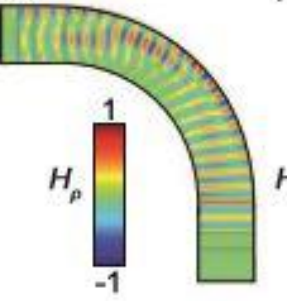

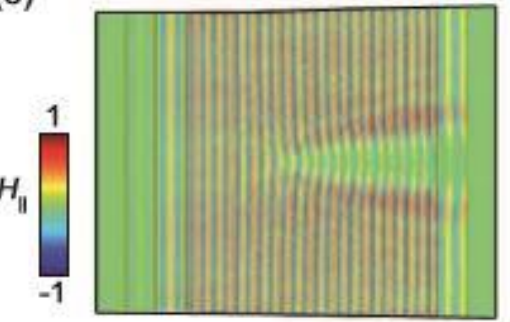

(f)

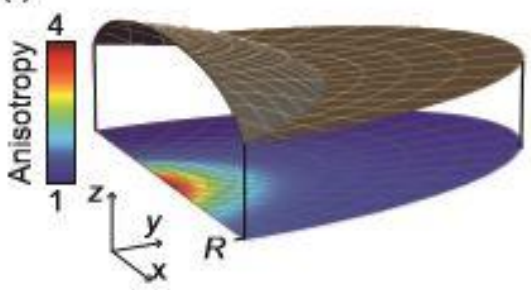

(g)

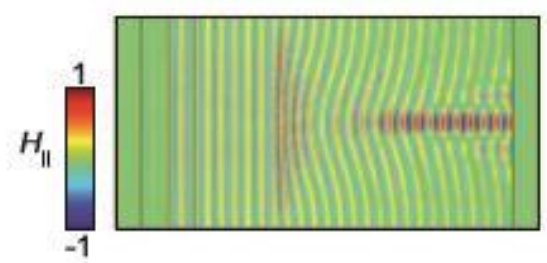

Figure 3. Demonstration and numerical verification of the versatility of the two-dimensional equivalence relations for a beam bender (a)-(c), beam splitter (d)-(e) and Möbius lens (f)-(g). Both the anisotropy (visualized by surface coloring on the symmetry plane of the waveguide) and the thickness variations of the core medium (visualized by the height of the floating surface representing the upper interface $\mathrm{z}=\tilde{\mathrm{a}}$ ) act on the in-plane magnetic fields to impose desired light flows and correspond to technologically feasible parameters. Without thickness variations (c), the in-plane magnetic fields cannot complete the bend.

We emphasize that our material implementation inside the core [Eq. (5)], which is based on the Helmholtz wave equation, is different from the straightforward application of transformation optics [Eq. (1)]. Our metamaterial is nonmagnetic and is not implemented outside of the waveguide core. Therefore, similar to the straightforward application of transformation optics, we exactly preserve the transverse wavevector $k_{2}$ and stretch the in-plane propagation $\tilde{\beta}=X \beta$. In contrast, we do not implement a metamaterial in the outer layers. Therefore, the Helmholtz equation will change the extinction coefficient $\tilde{\mathrm{k}}_{1}^{2}=\gamma(x, \mathrm{y}) \beta^{2}-\epsilon_{\text {out }} \omega^{2} / c^{2}$. To make sure that the profile is continuous at the interfaces, i.e., that the transverse profile propagates with a transformed propagation constant $\tilde{\beta}=\sqrt{\gamma(x, y)} \beta \approx \mathrm{X} \beta$ at the incident frequency $\omega$, we need to impose a geometry-dependent thickness variation

$$
\tilde{a}(x, y)=\frac{1}{k_{2}} \operatorname{atan}\left(\frac{\epsilon_{\text {core }}}{\epsilon_{\text {out }}} \sqrt{\gamma(x, y) \beta^{2}-\frac{\epsilon_{\text {out }} \omega^{2}}{c^{2}}} / k_{2}\right) .
$$

This thickness variation compensates for the lack of metamaterials outside of the waveguide. Our alternative equivalence relations [Eqs. (5)-(6)] lead to nonmagnetic metamaterial cores of varying thickness without need for metamaterial implementations in the outer layers.

\section{NONMAGNETIC METAMATERIAL WAVEGUIDE DEVICES}

We will present numerical simulations of three proof-of-principle devices, a beam bender based on a logarithmic map [37], a beam splitter based on a Schwarz-Christoffel transformation [51] and a conformal lens based on a Möbius transformation [37], to demonstrate the versatility and effectiveness of our equivalence relations. Figure 3 visualizes the symmetric thickness variation (the floating surface represents the thickness variation of the upper interface) and the anisotropy of the uniaxial metamaterial inside the core (color online on symmetry plane) for each waveguide component. The required metamaterial distributions and thickness variations are feasible and can be fabricated by 3D printing (direct laser writing) or other lithographic techniques [20-23]. Qualitatively, we observe that the in-plane magnetic fields in Figs. 3(b), 3(e), and $3(\mathrm{~g})$, bend, split and focus in a very efficient way. 


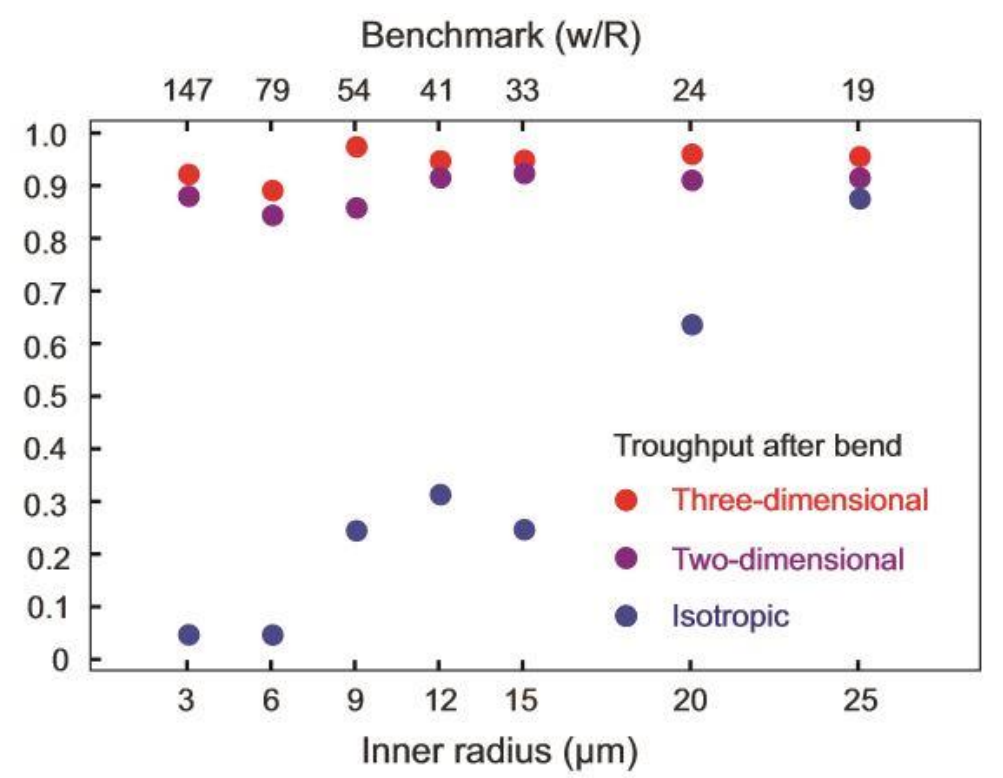

Figure 4. The throughput of our beam bender is evaluated for seven outer radii $\mathrm{R}$ at fixed beam width w. Increases in anisotropy are represented by the benchmark $\mathrm{w} / \mathrm{R}$, as a way to demonstrate the effectiveness of our equivalence relations (purple dots) compared to the conventional design of transformation optics (red dots) and an isotropic implementation with appropriate thickness variation (blue dots). The throughputs of our two-dimensional metamaterial cores are impressive, lying close to those of the three-dimensional implementation while isotropic metamaterial cores cannot maintain their performance as inner radii approach the free space wavelength $\lambda=1.5 \mu m$. Mesh convergence studies resulted in negligible error bars.

To quantitatively compare the performance of our two-dimensional designs to the bulky implementations of transformation optics, we performed several numerical simulations of beam bends with decreasing inner radius $\mathrm{R}$ while preserving the incident beam width w. As shown in Fig. 4, the throughput of our two-dimensional bends (from $84 \%$ to 93\%) is highly comparable to the three-dimensional implementations (from $86 \%$ to $95 \%$ ), even close to the subwavelength regime. In contrast, an isotropic metamaterial core supplemented by the required thickness variation cannot maintain high throughputs for small bending radii. Besides the anisotropy of metamaterial cores, the thickness variation is another crucial ingredient of the equivalence relations without which guided waves cannot complete the bend [Fig. 3(c)].

\section{CONCLUSION}

In summary, we have developed two-dimensional equivalence relations that allow for designing thin, nonmagnetic metamaterial waveguides of varying thickness. In this way, we present a versatile platform for controlled propagation of confined light using metamaterials with a pure dielectric response. Apart from the successful implementation of proof-ofprinciple optical components, e.g., beam benders, beam splitters and conformal lenses, our work introduces a novel paradigm towards holistic manipulation of guided modes: using metamaterial waveguides one may seamlessly split, bend and focus light in an integrated setup with minimized insertion loss [24-25].

\section{ACKNOWLEDGEMENTS}

S. V. and V. G. acknowledge fellowships from the Research Foundation Flanders (FWO-Vlaanderen). Work at VUB was also supported by BelSPO (Grant No. IAP P7-35 photonics@ be). 


\section{REFERENCES}

[1] J.B. Pendry, "Photonics: Metamaterials in the sunshine," Nature Materials 5, p. 599-600 (2006).

[2] N. Engheta and R. W. Ziolkowski, [Metamaterials: Physics and Engineering Explorations], Wiley, New York, (2006).

[3] C.M. Soukoulis and M. Wegener, "Past achievements and future challenges in the development of threedimensional photonic metamaterials," Nature Photonics 5, p. 523-530 (2011).

[4] X. Fan and I.M. White, "Optofluidic microsystems for chemical and biological analysis," Nature Photonics 5, p. 591-597 (2011).

[5] A. Alu and N. Engheta, "Dielectric sensing in $\epsilon$-near-zero narrow waveguide channels," Physical Review B 78, p. $045102(2008)$.

[6] M. Law, D.J. Sirbuly, J.C. Johnson, J. Goldberger, R.J. Saykally, and P. Yang, "Nanoribbon waveguides for subwavelength photonics integration," Science 305, p. 1269-1273 (2004).

[7] Y. Yang, A.Q. Liu, L.K. Chin, X.M. Zhang, D.P. Tsai, C.L. Lin, C. Lu, G.P. Wang, and N.I. Zheludev, "Optofluidic waveguide as a transformation optics device for lightwave bending and manipulation," Nature Communications 3, p. 651 (2012).

[8] D. Van Thourhout and J. Roels, "Optomechanical device actuation through the optical gradient force," Nature Photonics 4, p. 211-217 (2010).

[9] V. Ginis, P. Tassin, C.M. Soukoulis, and I. Veretennicoff, "Enhancing optical gradient forces with metamaterials," Physical Review Letters 110, p. 057401 (2013).

[10] J.D. Joannopoulos, S.G. Johnson, J.N. Winn, and R.D. Meade, [Photonic crystals: molding the flow of light], 2nd ed., New Jersey, Princeton University Press (2011).

[11] C. Caër, S. Combrié, X. Le Roux, E. Cassan, and A. De Rossi, "Extreme optical confinement in a slotted photonic crystal waveguide," Applied Physics Letters 105, p. 121111 (2014).

[12] S.P. Yu, J.D. Hood, J.A. Muniz, M.J. Martin, R. Norte, C.L. Hung, S.M. Meenehan, J.D. Cohen, O. Painter, and H.J. Kimble, "Nanowire photonic crystal waveguides for single-atom trapping and strong light-matter interactions," Applied Physics Letters 104, p. 111103 (2014).

[13] W.L. Barnes, A. Dereux, and T.W. Ebbesen, "Surface plasmon subwavelength optics," Nature 424, p. 824-830 (2003).

[14] D.K. Gramotnev, and S.I. Bozhevolnyi, "Nanofocusing of electromagnetic radiation," Nature Photonics 8, p. 1322 (2014).

[15] B. Dastmalchi, P. Tassin, T. Koschny, and C.M. Soukoulis, "A new perspective on plasmonics: confinement and propagation length of surface plasmons for different materials and geometries," Advanced Optical Materials 4, p. 177-184 (2016).

[16]P. Li, M. Lewin, A.V. Kretinin, J. Caldwell, K.S. Novoselov, T. Taniguchi, K. Watanabe, F. Gaussmann, and T. Taubner, "Hyperbolic phonon-polaritons in boron nitride for near-field optical imaging and focusing," Nature Communications 6, p. 8507 (2015).

[17] O. Takayama, D. Artigas, and L. Torner, "Lossless directional guiding of light in dielectric nanosheets using Dyakonov surface waves," Nature Nanotechnology 9, p. 419-424 (2014).

[18] S. Jahani and Z. Jacob, "All-dielectric metamaterials," Nature Nanotechnology 11, p. 23-36 (2016).

[19] S.J. Corbitt, M. Francoeur, and B. Raeymaekers, "Implementation of optical dielectric metamaterials: A review," Journal of Quantitative Spectroscopy and Radiative Transfer 158, p. 3-16 (2015).

[20] J. Fischer, T. Ergin, and M. Wegener, "Three-dimensional polarization-independent visible-frequency carpet invisibility cloak,” Optics Letters 36, p. 2059-2061 (2011).

[21]U. Zywietz, A.B. Evlyukhin, C. Reinhardt, and B.N. Chichkov, "Laser printing of silicon nanoparticles with resonant optical electric and magnetic responses," Nature Communications 5, p.3402 (2014).

[22] C.B. Arnold, P. Serra, and A. Piqué, "Laser direct-write techniques for printing of complex materials," MRS Bulletin 32, p. 23-31 (2007).

[23] P. Moitra, B.A. Slovick, W. Li, I.I. Kravchencko, D.P. Briggs, S. Krishnamurthy, and J. Valentine, "Large-scale all-dielectric metamaterial perfect reflectors," ACS Photonics 2, p. 692-698 (2015).

[24] S. Viaene, V. Ginis, J. Danckaert, and P. Tassin, "Transforming two-dimensional guided light using nonmagnetic metamaterial waveguides," Physical Review B 93, p. 085429 (2016).

[25] S. Viaene, V. Ginis, J. Danckaert, and P. Tassin, "Transforming guided waves with metamaterial waveguide cores," Proc. SPIE 9883, Metamaterials X, 98831F (April 18, 2016). 
[26] D. Schurig, J.J. Mock, B.J. Justice, S.A. Cummer, J.B. Pendry, A.F. Starr, and D.R. Smith, "Metamaterial electromagnetic cloak at microwave frequencies," Science 314, p. 977-980 (2006).

[27] U. Leonhardt, “Optical conformal mapping," Science 312, p. 1777-1780 (2006).

[28] B. Zhang, "Electrodynamics of transformation-based invisibility cloaking," Light: Science \& Applications 1, p. e32 (2012).

[29] A. Greenleaf, Y. Kurylev, M. Lassas, U. Leonhardt, and G. Uhlmann, "Cloaked electromagnetic, acoustic, and quantum amplifiers via transformation optics," Proceedings of the National Academy of Sciences 109, p. 1016910174 (2012).

[30] X. Ni, Z.J. Wong, M. Mrejen, Y. Wang, and X. Zhang, "An ultrathin invisibility skin cloak for visible light," Science 349, p. 1310-1314 (2015).

[31] M. Born and E. Wolf, [Principles of Optics: Electromagnetic Theory of Propagation, Interference and Diffraction of Light], Pergamon Press, New York, (1964).

[32] L.D. Landau and E.M. Lifshitz, [The classical theory of fields: Course of theoretical physics], 3rd ed., Pergamon Press, Oxford, (1971).

[33] S.M. Carroll, [Spacetime and geometry. An introduction to general relativity], Wiley, New York, (2004).

[34]F. de Felice, "On the gravitational field acting as an optical medium," General Relativity and Gravitation 2, p. 347-357 (1971).

[35] J. B. Pendry, A. Aubry, D.R. Smith, and S.A. Maier, "Transformation optics and subwavelength control of light," Science 337, p. 549-552 (2012).

[36] M. Rahm, S.A. Cummer, D. Schurig, J.B. Pendry, and D.R. Smith, "Optical design of reflectionless complex media by finite embedded coordinate transformations," Physical Review Letters 100, p. 063903 (2008).

[37] L. Xu and H. Chen, "Conformal transformation optics," Nature Photonics 9, p. 15-23 (2015).

[38] V. Ginis, J. Danckaert, I. Veretennicoff, and P. Tassin, "Controlling Cherenkov radiation with transformationoptical metamaterials," Physical Review Letters 113, p. 167402 (2014).

[39] P. Genevet, D. Wintz, A. Daniel, A. Ambrosio, A. She, R. Blanchard, and F. Capasso, "Controlled steering of Cherenkov surface plasmon wakes with a one-dimensional metamaterial," Nature Nanotechnology 10, p. 804809 (2015).

[40] F. Liu, and J. Li, "Gauge field optics with anisotropic media," Physical Review Letters 114, p. 103902 (2015).

[41] T. Ma, A.B. Khanikaev, S.H. Mousavi, and G. Shvets, "Guiding electromagnetic waves around sharp corners: topologically protected photonic transport in metawaveguides," Physical Review Letters 114, p. 127401 (2015).

[42] Editorial, "The power of analogies," Nature Photonics 8, p. 1 (2014).

[43] C. Sheng, H. Liu, Y. Wang, S.N. Zhu, and D.A. Genov, "Trapping light by mimicking gravitational lensing," Nature Photonics 7, p. 902-906 (2013).

[44] V. Ginis, P. Tassin, B. Craps, and I. Veretennicoff, "Frequency converter implementing an optical analogue of the cosmological redshift," Optics Express 18, p. 5350-5355 (2010).

[45] Y. Liu, T. Zentgraf, G. Bartal, and X. Zhang, "Transformational plasmon optics," Nano Letters 10, p. 1991-1997 (2010).

[46]P.A. Huidobro, M.L. Nesterov, L. Martin-Moreno, and F.J. Garcia-Vidal, "Transformation optics for plasmonics," Nano Letters 10, p. 1985-1990 (2010).

[47] M. Kadic, S. Guenneau, S. Enoch, P.A. Huidobro, L. Martin-Moreno, F.J. Garcia-Vidal, J. Renger, and R. Quidant, "Transformation plasmonics," Nanophotonics 1, p. 51-64 (2012).

[48] I.I. Smolyaninov, V.N. Smolyaninova, A.V. Kildishev, and V.M. Shalaev,"Anisotropic metamaterials emulated by tapered waveguides: application to optical cloaking," Physical Review Letters 102, p. 213901 (2009).

[49] A. Vakil and N. Engheta, "Transformation optics using graphene," Science 332, p. 1291-1294 (2011).

[50] P.A. Huidobro, M. Kraft, R. Kun, S.A. Maier and J.B. Pendry, "Graphene, plasmons and transformation optics," Journal of Optics 18, p. 044024 (2016).

[51] R. Schinzinger and P.A.A. Laura, [Conformal Mapping: Methods and Applications], Dover Publications, New York, (2003). 